[7] C. Wen and D. J. Hill, "Global boundedness of discrete-time adaptive control just using estimator projection," Automatica, pp. 1143-1157, Nov. 1992.

[8] R. H. Middleton and Y. Wang, "The internal model principle in the adaptive control of time varying linear systems," Tech. Rep. EE8830, Univ. Newcastle, Australia, 1988.

[9] B. Egardt, Stability of Adaptive Controllers. New York: SpringerVerlag, 1979.

[10] G. C. Goodwin and K. S. Sin, Adaptive Filtering Prediction and Control. Englewood Cliffs, NJ: Prentice-Hall, 1984.

[11] R. H. Middleton and G. C. Goodwin, "Adaptive control of time varying linear systems," IEEE Trans. Automat. Contr., vol. 33, pp. 150-155, 1988.

[12] B. E. Ydstie, "Stability of discrete model reference control revisited," Syst. Contr. Lett., vol. 13, pp. 429-438, 1989.

[13] , "Stability of the direct self-tuning regulator," in Foundations of Adaptive Control, P. V. Kokotovic, Ed., 1991, pp. 201-238.

[14] S. M. Naik, P. R. Kumar, and B. E. Ydstie, "Robust continuous-time adaptive control by parameter projection," IEEE Trans. Automat. Contr. vol. 37, pp. 182-197, 1992.

[15] H. Minc, Nonnegative Matrices. New York: Wiley, 1988.

\section{Robust Stability of Discrete-Time Systems Under Parametric Perturbations}

Mehmet Karan, M. Erol Sezer, and Ogan Ocali

Abstract-Stability robustness analysis of a system under parametric perturbations is concerned with characterizing a region in the parameter space in which the system remains stable. In this paper, two methods are presented to estimate the stability robustness region of a linear, time-invariant, discrete-time system under multiparameter additive perturbations. An inherent difficulty, which originates from the nonlinear appearance of the perturbation parameters in the inequalities defining the robustness region, is resolved by transforming the problem to stability of a higher order continuous-time system. This allows for application of the available results on stability robustness of continuous-time systems to discrete-time systems. The results are also applied to stability analysis of discrete-time interconnected systems, where the interconnections are treated as perturbations on decoupled stable subsystems.

\section{INTRODUCTION}

An essential feature of complex dynamic systems is the uncertainty in the system parameters, which may arise due to modeling errors or change of operating conditions. The analysis of stability in the presence of uncertainty is the subject of the robust stability problem. A common approach to stability robustness analysis is to model the uncertainty as perturbations on a nominal stable model. A measure of degree of stability of the nominal system can then be used to obtain bounds on the perturbations which the system can tolerate without going unstable.

Lyapunov's direct method provides a convenient way to estimate the degree of stability. It also directly yields bounds on tolerable perturbations [1]. This feature of the Lyapunov approach has been used

Manuscript received January 4, 1991; revised June 7, 1991 and April 16, 1993.

M. Karan is with the Department of Systems Engineering, Australian National University, ACT 0200, Australia.

M. E. Sezer and O. Ocali are with the Department of Electrical and Electronics Engineering, Bilkent University, Ankara 06533, Turkey.

IEEE Log Number 9216453 among many others in [2]-[5] to obtain explicit robustness bounds for state-space models of continuous-time systems under additive perturbations. Some of these results have also been reproduced for discrete-time systems (see, for example [6]-[8])

The main objective of this paper is to link the stability robustness problem of discrete-time systems to that of continuous-time systems. We show, using two different approaches, that stability robustness of a discrete-time system can be reformulated as that of an auxiliary continuous-time system. One of these approaches makes use of Lyapunov theory and yields a sufficient condition. The second approach, which is based on the properties of Kronecker products, provides a necessary and sufficient condition at the expense of an increase in the dimensionality. This is a pleasing development, since it allows for a direct application of the known results on stability robustness bounds for continuous-time systems to discrete-time systems. The results are applied to stability analysis of interconnected systems, where the interconnections are treated as perturbations on a collection of stable subsystems. This demonstrates how a knowledge of the structure of perturbations can be exploited to obtain simple robustness bounds.

\section{Problem Statement}

Consider a discrete-time system under additive multiparameter perturbations, which is described as

$$
\mathcal{D}: x(k+1)=A(p) x(k)
$$

where $x(k) \in \mathcal{R}^{n}$ is the state of $\mathcal{D}$ at the discrete time instant $k \in \mathcal{Z}_{+}, p=\left[\begin{array}{llll}p_{1} & p_{2} & \cdots & p_{m}\end{array}\right]^{T} \in \mathcal{R}^{m}$ is a vector of real perturbation parameters, and

$$
A(p)=A+\sum_{r=1}^{m} p_{r} E_{r}
$$

with $A$ and $E_{r}, r=1,2, \cdots, m$, being constant $n \times n$ real matrices We assume that the matrix $A(0)=A$ is Schur-stable, that is, has all the eigenvalues in the open unit disk in the complex plane.

We would like to describe an open neighborhood of the origin in the parameter space in which $\mathcal{D}$ remains stable. More precisely, we are interested in a region

$$
\Omega=\{p \mid A(\alpha p) \text { is Schur-stable for all } \alpha \in[0,1]\}
$$

in the parameter space $\mathcal{R}^{m}$. Since, in general, it is difficult to characterize $\Omega$ explicitly in terms of the perturbation parameters, we aim at obtaining estimates of $\Omega$ as regular volumes embedded in $\Omega$ which can be characterized explicitly.

\section{Estimation of Robustness Region Via Lyapunov Theory}

Our first approach to estimating $\Omega$ is through Lyapunov theory. Let $V(x)=x^{T} P x$ be a Lyapunov function for the nominal system corresponding to $p=0$, where $P \in \mathcal{R}^{n \times n}$ is the unique, symmetric, positive-definite solution of the discrete-time Lyapunov equation

$$
A^{T} P A-P=-Q
$$

for some symmetric, positive-definite matrix $Q \in \mathcal{R}^{n \times n}$. The difference of $V(x)$ along the solutions of the perturbed system $\mathcal{D}$ in (2.1) is computed as

$$
\left.\Delta V(x(k))\right|_{\mathcal{D}_{p}}=x^{T}(k) Q^{1 / 2}[Q(p)-I] Q^{1 / 2} x(k)
$$


where

$$
\begin{aligned}
Q(p)= & \sum_{r=1}^{m} p_{r} Q^{-1 / 2}\left(E_{r}^{T} P A+A^{T} P E_{r}\right) Q^{-1 / 2} \\
& +\sum_{r=1}^{m} \sum_{s=1}^{m} p_{r} p_{s} Q^{-1 / 2} E_{r}^{T} P E_{s} Q^{-1 / 2}
\end{aligned}
$$

From (3.2), we observe that a sufficient condition for $p \in \Omega$ is that $Q(p)-I$ be negative definite or, equivalently,

$$
\sigma_{M}[Q(p)]<1
$$

where $\sigma_{M}(\cdot)$ denotes the maximum singular value of the indicated matrix. For the single-parameter perturbation case, i.e., when $m=1$, (3.4) can be reduced, using a majorization on $\sigma_{M}[Q(p)]$, to a quadratic inequality in $|p|$, from which an explicit bound for $|p|$ can be obtained. In the multiparameter perturbation case $(m>1)$, however, the left-hand side of (3.4) becomes a quadratic polynomial in $\left\{\left|p_{r}\right|\right\}$, and characterization of $\Omega$ in terms of $\left|p_{r}\right|$ is not as easy. The only available result $[8]$ in this case involves the calculation of singular values of an $m n \times m n$ matrix.

To avoid the difficulty, we try to obtain an alternative to the condition in (3.4), which guarantees negative definiteness of $Q(p)-I$, and thus stability of $\mathcal{D}$. For this purpose, we observe that

$$
\operatorname{det}[Q(p)-I]=-1^{n} \operatorname{det} \bar{F}(p)
$$

where

$$
\bar{F}(p)=-I+\sum_{r=1}^{m} p_{r} \bar{G}_{r}
$$

with

$$
\bar{G}_{r}=\left[\begin{array}{cc}
0 & P^{1 / 2} E_{r} Q^{-1 / 2} \\
Q^{-1 / 2} E_{r}^{T} P^{1 / 2} & Q^{-1 / 2}\left(E_{r}^{T} P A+A^{T} P E_{r}\right) Q^{-1 / 2}
\end{array}\right]
$$

We now consider an auxiliary continuous-time system

$$
\overline{\mathcal{S}}: \dot{\bar{\xi}}(t)=\bar{F}(p) \bar{\xi}(t)
$$

for which we define a stability region around the origin, similar to $\Omega$ in (2.3), as

$$
\bar{\Omega}=\{p \mid \bar{F}(\alpha p) \text { is Hurwitz-stable for all } \alpha \in[0,1]\}
$$

and state the following.

Theorem 1: $\bar{\Omega} \subset \Omega$.

Proof: Fix $p^{*} \in \bar{\Omega}$, and note that: i) $Q\left(\alpha p^{*}\right)-I$ is symmetric with all real eigenvalues, ii) eigenvalues of $Q\left(\alpha p^{*}\right)-I$ are continuous in $\alpha$, and iii) $Q(0)-I=-I$ is negative definite. Now, if $Q\left(\alpha p^{*}\right)-I$ is negative definite for all $\alpha>0$, then $p^{*} \in \Omega$. Otherwise, there exists $\alpha^{*}>0$ such that $Q\left(\alpha p^{*}\right)-I$ is negative definite for all $0 \leq \alpha<\alpha^{*}$, and $\operatorname{det}\left[Q\left(\alpha^{*} p^{*}\right)-I\right]=0$. Then, by (3.5), $\bar{F}\left(\alpha^{*} p^{*}\right)$ has an eigenvalue at the origin, which implies that $\alpha^{*} p^{*} \notin \bar{\Omega}$. Thus, $\alpha^{*}>1$, and again, $p^{*} \in \Omega$.

Theorem 1 is an attractive result because it constructs a link between stability robustness analysis of discrete- and continuous-time systems. For example, choosing $v(\bar{\xi})=\bar{\xi}^{T} \bar{\xi}$ as a Lyapunov function for $\overline{\mathcal{S}}$, it can easily be shown that $\overline{\mathcal{S}}$, and therefore $\mathcal{D}$, is stable if

$$
\sigma_{M}\left(\sum_{r=1}^{m} p_{r} \bar{G}_{r}\right)<1
$$

Using the majorizations employed in [5], it is possible to estimate diamond, parallelepiped, and sphere-shaped estimates of the robustness region $\Omega$ in the parameter space, as we state with the following corollary.
Corollary 1: The discrete-time perturbed system $\mathcal{D}$ is stable if the following bounds are satisfied

$$
\begin{aligned}
& \bar{\Omega}_{D}: \sum_{r=1}^{m}\left|p_{r}\right| \sigma_{M}\left(\bar{G}_{r}\right)<1 \\
& \bar{\Omega}_{P}: \max _{1 \leq r \leq m}\left|p_{r}\right|<\sigma_{M}^{-1}\left(\sum_{r=1}^{m}\left|\bar{G}_{r}\right|\right) \\
& \bar{\Omega}_{S}:\left(\sum_{r=1}^{m} p_{r}^{2}\right)^{1 / 2}<\sigma_{M}^{-1 / 2}\left(\sum_{r=1}^{m} \bar{G}_{r}^{T} \bar{G}_{r}\right) .
\end{aligned}
$$

\section{Direct Estimation of the Stabllity Region}

Our second approach to estimating the stability region $\Omega$ is a direct one based on the properties of Kronecker products [9].

Let us define

$$
\begin{aligned}
\tilde{F}(p) & =\left[\begin{array}{cc}
-I & I \otimes A(p) \\
A(p) \otimes I & -I
\end{array}\right] \\
& =\tilde{F}+\sum_{r=1}^{m} p_{r} \tilde{G}_{r}
\end{aligned}
$$

where

$$
\tilde{F}=\left[\begin{array}{cc}
-I & I \otimes A \\
A \otimes I & -I
\end{array}\right]
$$

and

$$
\tilde{G}_{r}=\left[\begin{array}{cc}
0 & I \otimes E_{r} \\
E_{r} \otimes I & 0
\end{array}\right] .
$$

As in the previous section, we associate a continuous-time system with $\tilde{F}(p)$

$$
\tilde{\mathcal{S}}: \dot{\tilde{\xi}}(t)=\tilde{F}(p) \tilde{\xi}(t)
$$

and define

$$
\tilde{\Omega}=\{p \mid \tilde{F}(\alpha p) \text { is Hurwitz-stable for all } \alpha \in[0,1]\} .
$$

We then have the following.

Theorem 2: $\tilde{\Omega}=\Omega$.

Proof: Using the properties of Kronecker products, we have

$$
\operatorname{det}[s I-\tilde{F}(p)]=\operatorname{det}\left[(s+1)^{2} I-A(p) \otimes A(p)\right]
$$

so that the eigenvalues of $\tilde{F}(p)$ and those of $A(p) \otimes A(p)$ are related as

$$
\lambda\{\tilde{F}(p)\}=-1+\lambda^{1 / 2}\{A(p) \otimes A(p)\} .
$$

$\Omega \subset \tilde{\Omega}$ follows from (4.7) on noting that, when $A(p)$ is Schur-stable, then eigenvalues of $A(p) \otimes A(p)$ are all within the unit circle. To prove that $\tilde{\Omega} \subset \Omega$, fix $p^{*} \in \tilde{\Omega}$. If $A\left(\alpha p^{*}\right)$ is Schur-stable for all $\alpha>0$, then $p^{*} \in \Omega$. Otherwise, there exists $\alpha^{*}>0$ such that $A\left(\alpha p^{*}\right)$ is Schur-stable for all $0 \leq \alpha<\alpha^{*}$, and $A\left(\alpha^{*} p^{*}\right)$ has an eigenvalue on the unit circle. Then $A\left(\alpha^{*} p^{*}\right) \otimes A\left(\alpha^{*} p^{*}\right)$ has an eigenvalue at $s=1$, and by (4.7), $\tilde{F}\left(\alpha^{*} p^{*}\right)$ has an eigenvalue at $s=0$. Thus, $\alpha^{*}>1$, and therefore, $p^{*} \in \Omega$. This completes the proof.

Like Theorem 1, Theorem 2 also allows us to use continuoustime results to obtain stability robustness bounds for $\mathcal{D}$. Moreover, since it is directly based on the eigenvalues of $A(p)$, it provides a necessary and sufficient condition for stability of $\mathcal{D}$. However, since the dimension of the auxiliary system $\tilde{\mathcal{S}}$ is higher than that of $\overline{\mathcal{S}}$, further majorizations involved in the estimation of $\tilde{\Omega}$ may result in more conservative robustness bounds than Theorem 1 . 
Although standard Lyapunov theory can be used to obtain perturbation bounds for $\tilde{\mathcal{S}}$, since the nominal system matrix $\tilde{F}$ of $\tilde{\mathcal{S}}$ is not a simple one as that of $\overline{\mathcal{S}}$, we would like to describe a method to generate a suitable Lyapunov function for $\tilde{F}$.

Lemma: Let $A_{1}$ and $A_{2}$ be Schur-stable matrices, for which there exist positive-definite matrices $P_{1}$ and $P_{2}$ that satisfy

$$
A_{i}^{T} P_{i} A_{i}-P_{i}=-Q_{i}, \quad i=1,2
$$

for some positive-definite matrices $Q_{1}$ and $Q_{2}$. Then, the matrix

$$
\hat{F}=\left[\begin{array}{cc}
-I & I \otimes A_{2} \\
A_{1} \otimes I & -I
\end{array}\right]
$$

is Hurwitz-stable, and

$$
\hat{P}=\left[\begin{array}{cc}
P_{1} \otimes P_{2} & 0 \\
0 & P_{1} \otimes P_{2}
\end{array}\right]
$$

is a Lyapunov matrix for $\hat{F}$ which satisfies

$$
\hat{F}^{T} \hat{P}+\hat{P} \hat{F}=-\hat{Q}-\hat{F}^{T} \hat{P} \hat{F}
$$

where

$$
\hat{Q}=\left[\begin{array}{cc}
Q_{1} \otimes P_{2} & 0 \\
0 & P_{1} \otimes Q_{2}
\end{array}\right]
$$

Proof: Follows directly from

$$
\hat{F}^{T} \hat{P}+\hat{P} \hat{F}=(\hat{F}+I)^{T} \hat{P}(\hat{F}+I)-\hat{P}-\hat{F}^{T} \hat{P} \hat{F}
$$

on noting that $(\hat{F}+I)^{T} \hat{P}(\hat{F}+I)-\hat{P}=-\hat{Q}$.

Applying the result of the Lemma to $\tilde{F}$ in (4.2) with $Q_{1}=Q_{2}=I$, we obtain

$$
\tilde{P}=\left[\begin{array}{cc}
P \odot P & 0 \\
0 & P \odot P
\end{array}\right], \quad \tilde{Q}=\left[\begin{array}{cc}
I \otimes P & 0 \\
0 & P \otimes I
\end{array}\right]
$$

where $P$ is the solution of

$$
A^{T} P A-P=-I \text {. }
$$

Now, choosing $v(\tilde{\xi})=\tilde{\xi}^{T} \tilde{P} \tilde{\xi}$ as a Lyapunov function for $\tilde{\mathcal{S}}$ of (4.4), and using the result of [10], we obtain the following perturbation bounds for stability.

Corollary 2: The discrete-time perturbed system $\mathcal{D}$ is stable if the following bounds are satisfied:

$$
\begin{aligned}
& \tilde{\Omega}_{D}: \sum_{r=1}^{m}\left|p_{r}\right| \sigma_{M}\left(\tilde{H}_{r}\right)<1 \\
& \tilde{\Omega}_{P}: \max _{1 \leq r \leq m}\left|p_{r}\right|<\sigma_{M}^{-1}\left(\sum_{r=1}^{m}\left|\tilde{H}_{r}\right|\right) \\
& \tilde{\Omega}_{S}:\left(\sum_{r=1}^{m} p_{r}^{2}\right)^{1 / 2}<\sigma_{M}^{-1 / 2}\left(\sum_{r=1}^{m} \tilde{H}_{r}^{T} \tilde{H}_{r}\right)
\end{aligned}
$$

where (see (4.17) at the bottom of the page).

\section{APPliCATION to INTERCONNECTED SystemS}

Consider a discrete-time interconnected system which consists of $N$ subsystems described as

$\mathcal{D}: x_{i}(k+1)=A_{i} x_{i}(k)+\sum_{j=1}^{N} p_{i j} A_{i j} x_{j}(k)$,

$$
i=1,2, \cdots, N
$$

where $x_{i}(k) \in \mathcal{R}^{n_{i}}$ is the state of the $i$ th isolated subsystem

$$
\mathcal{D}_{i}: x_{i}(k+1)=A_{i} x_{i}(k), \quad i=1,2, \cdots, N
$$

which is assumed to be stable, $A_{i j}$ are constant real matrices, and $p_{i j}$ are interconnection gains, which are treated as perturbation parameters.

Letting $x=\left[\begin{array}{llll}x_{1}^{T} & x_{2}^{T} & \cdots & x_{N}^{T}\end{array}\right]^{T}, A=\operatorname{diag}\left\{A_{1}, A_{2}, \cdots, A_{N}\right\}$ and $E_{i j}=\left(E_{p q}^{i j}\right)_{N \times N}$, with

$$
E_{p q}^{i j}= \begin{cases}A_{i j}, & p=i, q=j \\ 0, & \text { otherwise }\end{cases}
$$

the interconnected system in (5.1) can be described in a compact form as

$$
\mathcal{D}: x(k+1)=\left(A+\sum_{i=1}^{N} \sum_{j=1}^{N} p_{i j} E_{i j}\right) x(k) .
$$

Choosing $V(x)=x^{T} P x$ as a Lyapunov function for $\mathcal{D}$, where $P$ is the solution of (3.1) with $Q=I$, Corollary 1 gives the following robustness regions in the parameter space of $\mathcal{D}$ :

$$
\begin{aligned}
& \bar{\Omega}_{D}: \sum_{i=1}^{N} \sum_{j=1}^{N}\left|p_{i j}\right| \sigma_{M}\left(\bar{G}_{i j}\right)<1 \\
& \bar{\Omega}_{P}: \max _{i, j}\left|p_{i j}\right|<\sigma_{M}^{-1}\left(\sum_{i=1}^{N} \sum_{j=1}^{N}\left|\bar{G}_{i j}\right|\right) \\
& \bar{\Omega}_{S}: \sum_{i=1}^{N} \sum_{j=1}^{N} p_{i j}^{2}<\sigma_{M}^{-1 / 2}\left(\sum_{i=1}^{N} \sum_{j=1}^{N} \bar{G}_{i j}^{T} \bar{G}_{i j}\right)
\end{aligned}
$$

where

$$
\bar{G}_{i j}=\left[\begin{array}{cc}
0 & P^{1 / 2} E_{i j} \\
E_{i j}^{T} P^{1 / 2} & E_{i j}^{T} P A+A^{T} P E_{i j}
\end{array}\right] .
$$

From the block-diagonal structure of $A$, it follows that $P=$ $\operatorname{diag}\left\{P_{1}, P_{2}, \cdots, P_{N}\right\}$, where $P_{i}$ are solutions of

$$
A_{i}^{T} P_{i} A_{i}-P_{i}=-I \text {. }
$$

This block-diagonal structure of $P$, together with the special structures of the perturbation matrices $E_{i j}$, allows for obtaining explicit expressions for $\sigma_{M}\left(\bar{G}_{i j}\right)$ appearing in (5.5). For example,

$$
\sigma_{M}\left(\bar{G}_{i j}\right)=\sigma_{M}\left[\begin{array}{cc}
0 & G_{i j} \\
G_{i j}^{T} & 0
\end{array}\right]
$$

where $G_{i j}^{T}=\left[A_{i j}^{T} P_{i}^{1 / 2} \quad A_{i j}^{T} P_{i} A_{i}\right]$, so that

$$
\sigma_{M}\left(\bar{G}_{i j}\right)=\sigma_{M}\left(G_{i j}\right) \leq \sigma_{M}^{1 / 2}\left(A_{i j}^{T} P_{i} A_{i j}\right) \sigma_{M}^{1 / 2}\left(P_{i}\right) .
$$

Thus, the stability region $\bar{\Omega}_{D}$ in (5.5) includes the region

$$
\bar{\Omega}_{D}^{\prime}: \sum_{i=1}^{N} \sum_{j=1}^{N}\left|p_{i j}\right| \sigma_{M}^{1 / 2}\left(P_{i}\right) \sigma_{M}^{1 / 2}\left(A_{i j}^{T} P_{i} A_{i j}\right)<1
$$

$$
\tilde{H}_{r}=\left[\begin{array}{cc}
0 & P^{1 / 2} @ P^{1 / 2} E_{r}+E_{r}^{T} P^{1 / 2} \otimes P^{1 / 2} \\
P^{1 / 2} @ E_{r}^{T} P^{1 / 2}+P^{1 / 2} E_{r} \otimes P^{1 / 2} & 0
\end{array}\right]
$$


which is smaller than $\bar{\Omega}_{D}$, but easier to compute. Note that it is also possible to give an explicit expression for $\sigma_{M}^{-1 / 2}\left(\sum_{i} \sum_{j} \bar{G}_{i j}^{T} \bar{G}_{i j}\right)$ to obtain an approximation to $\bar{\Omega}_{S}$ in (5.5).

Alternatively, applying Corollary 2 to the interconnected system $\mathcal{D}$ in (5.1), we get the robustness regions

$$
\begin{aligned}
& \tilde{\Omega}_{D}: \sum_{i=1}^{N} \sum_{j=1}^{N}\left|p_{i j}\right| \sigma_{M}\left(\bar{H}_{i j}\right)<1 \\
& \tilde{\Omega}_{P}: \max _{i, j}\left|p_{i j}\right|<\sigma_{M}^{-1}\left(\sum_{i=1}^{N} \sum_{j=1}^{N}\left|\bar{H}_{i j}\right|\right) \\
& \tilde{\Omega}_{S}:\left(\sum_{i=1}^{N} \sum_{j=1}^{N} p_{i j}^{2}\right)^{1 / 2}<\sigma_{M}^{-1 / 2}\left(\sum_{i=1}^{N} \sum_{j=1}^{N} \bar{H}_{i j}^{T} \bar{H}_{i j}\right)
\end{aligned}
$$

where (see (5.12) at the bottom of the page). Although it is not easy to express the coefficients of $\left|p_{i j}\right|$ in (5.11) directly in terms of $\boldsymbol{A}_{i j}$ and $P_{i}, \tilde{H}_{i j}$ are nevertheless sparse matrices, and finding the regions in (5.11) does not require excessive computational effort.

Before closing the section, we would like to compare the estimates $\bar{\Omega}_{D}$ and $\bar{\Omega}_{D}^{\prime}$ in (5.5) and (5.10) of the robustness region with that obtained by the composite Lyapunov function approach of [10]. Following their approach, it can be shown that $\mathcal{D}$ is stable if the aggregate matrix $W=\left(w_{i j}\right)_{N \times N}$ is an $M$-matrix [11], where

$$
\begin{aligned}
w_{i j}=\left\{\sigma_{M}^{1 / 2}\left(P_{i}\right)-\left[\sigma_{M}\left(P_{i}\right)-1\right]^{1 / 2}\right\} \delta_{i j} & \\
& -\left|p_{i j}\right| \sigma_{M}^{1 / 2}\left(A_{i j}^{T} P_{i} A_{i j}\right)
\end{aligned}
$$

with $P_{i}$ being the solution of (5.7). At this point, an estimate $\Omega^{W}$ of the stability region can be characterized by a set of inequalities resulting from the $M$-matrix conditions. However, since $\left|p_{i j}\right|$ appear nonlinearly in these inequalities, it is not possible to compare $\Omega^{W}$ with $\bar{\Omega}_{D}$ in (5.5) or $\bar{\Omega}_{D}^{\prime}$ in (3.10).

To obtain an explicit characterization of $\Omega^{W}$, we note that $W$ is an $M$-matrix if

$$
\sigma_{M}\left(\sum_{i=1}^{N} \sum_{j=1}^{N}\left|p_{i j}\right| \hat{W}_{i j}\right)<1
$$

where $\hat{W}_{i j}$ has a single nonzero element in the $(i, j)$ th position given by

$$
\begin{aligned}
& \frac{\sigma_{M}^{1 / 2}\left(A_{i j}^{T} P_{i} A_{i j}\right)}{\sigma_{M}^{1 / 2}\left(P_{i}\right)-\left[\sigma_{M}\left(P_{i}\right)-1\right]^{1 / 2}} \\
& =\sigma_{M}^{1 / 2}\left(A_{i j}^{T} P_{i} A_{i j}\right)\left\{\sigma_{M}^{1 / 2}\left(P_{i}\right)+\left[\sigma_{m}\left(P_{i}\right)-1\right]^{1 / 2}\right\}
\end{aligned}
$$

From (5.14), an estimate of $\Omega^{W}$ is obtained as

$$
\begin{aligned}
& \Omega_{D}^{W}: \sum_{i=1}^{N} \sum_{j=1}^{N}\left|p_{i j}\right| \sigma_{M}^{1 / 2}\left(A_{i j}^{T} P_{i} A_{i j}\right) \\
& \cdot\left\{\sigma_{M}^{1 / 2}\left(P_{i}\right)+\left[\sigma_{M}\left(P_{i}\right)-1\right]^{1 / 2}\right\}<1 .
\end{aligned}
$$

Note that the estimate in (5.16) is the same as the region one would obtain by maximizing $\left|p_{i j}\right|$ using the method of [12]. From (5.16), it is clear that

$$
\Omega_{D}^{W} \subset \bar{\Omega}_{D}^{\prime} \subset \bar{\Omega}_{D}
$$

Although composite Lyapunov functions are known [10] to yield less conservative robustness bounds than ordinary Lyapunov functions for systems with structured perturbations, (5.17) claims the opposite. Obviously, the reason is that the whole advantage of using the $M$-matrix conditions on the aggregate matrix $W$ is lost at the majorization step leading to (5.14). This is demonstrated by an example in the next section.

\section{EXAMPLE}

To illustrate the application of our results, we consider an interconnection of three subsystems, with

$$
\begin{gathered}
A_{1}=\left[\begin{array}{cc}
-0.25 & 0 \\
0.5 & 0.75
\end{array}\right], \quad A_{2}=\left[\begin{array}{cc}
0.5 & 1 \\
0 & 0.25
\end{array}\right], \\
A_{3}=\left[\begin{array}{cc}
0.25 & 0 \\
0 & -0.25
\end{array}\right] \\
A_{12}=\left[\begin{array}{ll}
0 & 1 \\
0 & 0
\end{array}\right], \quad A_{21}=\left[\begin{array}{ll}
0 & 1 \\
1 & 0
\end{array}\right], \quad A_{32}=\left[\begin{array}{ll}
1 & 0 \\
1 & 0
\end{array}\right]
\end{gathered}
$$

and all other interconnection matrices being zero. Note that $\mathcal{D}$ has a block-triangular structure, i.e., the third subsystem does not form a loop with the other two. This structure for $\mathcal{D}$ is chosen purposefully to provide a comparison of the estimates of robustness regions obtained by different methods. Otherwise, an interconnection of only the first two subsystems would be sufficient to illustrate our result.

The exact region of stability can be obtained from the characteristic polynomial of the closed-loop system matrix as

$$
\Omega:-1.0625<p_{12} p_{21}<0.9375 .
$$

Note that $\Omega$ is independent of $p_{32}$, as expected from the blocktriangular structure of $\mathcal{D}$.

We calculate from $(5.7)$

$$
\begin{aligned}
& P_{1}=\left[\begin{array}{ll}
1.484 & 0.722 \\
0.722 & 2.286
\end{array}\right], P_{2}=\left[\begin{array}{ll}
1.333 & 0.762 \\
0.762 & 2.895
\end{array}\right], \\
& P_{3}=\left[\begin{array}{cc}
1.067 & 0 \\
0 & 1.067
\end{array}\right]
\end{aligned}
$$

and obtain the robustness regions

$$
\begin{gathered}
\bar{\Omega}_{D}: 1.333\left|p_{12}\right|+2.829\left|p_{21}\right|+1.509\left|p_{32}\right|<1 \\
\bar{\Omega}_{P}: \max \left\{\left|p_{12}\right|,\left|p_{21}\right|,\left|p_{32}\right|\right\}<0.302 \\
\bar{\Omega}_{S}:\left(p_{12}^{2}+p_{21}^{2}+p_{32}^{2}\right)^{1 / 2}<0.329
\end{gathered}
$$

from (5.5). A further majorization as in (5.10) yields

$$
\bar{\Omega}_{D}^{\prime}: 2.005\left|p_{12}\right|+3.205\left|p_{21}\right|+1.509\left|p_{32}\right|<1 .
$$

Alternatively, (5.11) produces the bounds

$$
\begin{gathered}
\tilde{\Omega}_{D}: 2.751\left|p_{12}\right|+4.210\left|p_{21}\right|+2.712\left|p_{32}\right|<1 \\
\tilde{\Omega}_{P}: \max \left\{\left|p_{12}\right|,\left|p_{21}\right|,\left|p_{32}\right|\right\}<0.202 \\
\tilde{\Omega}_{S}:\left(p_{12}^{2}+p_{21}^{2}+p_{32}^{2}\right)^{1 / 2}<0.202 .
\end{gathered}
$$

Note that the bounds in (6.7) are quite worse than those in (6.5), although they are based on a stronger result. Apparently, $v(\tilde{\xi})=$ $\tilde{\xi}^{T} \tilde{P} \tilde{\xi}$ with $\tilde{P}$ as in (4.14) is not the best Lyapunov function for $\tilde{\mathcal{S}}$ of (4.4).

$$
\tilde{H}_{i j}=\left[\begin{array}{cc}
0 & P^{1 / 2} \otimes P^{1 / 2} E_{i j}+E_{i j}^{T} P^{1 / 2} \otimes P^{1 / 2} \\
P^{1 / 2} \otimes E_{i j}^{T} P^{1 / 2}+P^{1 / 2} E_{i j} \otimes P^{1 / 2} & 0
\end{array}\right] .
$$


Application of the results of $[8]$ yields the bounds

$$
\begin{gathered}
\Omega_{P}: \max \left\{\left|p_{12}\right|,\left|p_{21}\right|,\left|p_{32}\right|\right\}<0.212 \\
\Omega_{S}:\left[\left(p_{12} \mp 0.085\right)^{2}+\left(p_{21} \mp 0.352\right)^{2}\right. \\
\left.+\left(p_{32} \mp 0.059\right)^{2}\right]^{1 / 2}<0.668
\end{gathered}
$$

where the constants in $\Omega_{S}$ have the same sign as the corresponding parameters. Note that [8] has no counterpart of $\bar{\Omega}_{D}$ and $\Omega_{P}$ is inferior to $\bar{\Omega}_{P}$. Also, the largest sphere with its center at the origin which is included in $\Omega_{S}$ is given by

$$
\Omega_{S}^{\prime}:\left(p_{12}^{2}+p_{21}^{2}+p_{32}^{2}\right)^{1 / 2}<0.308
$$

and is smaller than $\bar{\Omega}_{S}$.

On the other hand, the composite Lyapunov function approach of [10] yields the aggregate matrix

$$
W=\left[\begin{array}{ccc}
1 & -3.598\left|p_{12}\right| & 0 \\
-5.864\left|p_{21}\right| & 1 & 0 \\
0 & -1.886\left|p_{32}\right| & 1
\end{array}\right] .
$$

Note that the block-triangular structure of $\mathcal{D}$ is reflected in the structure of $W$. The robustness region is specified by the $M$-matrix conditions on $W$ as

$$
\Omega^{W}:\left|p_{12} p_{21}\right|<0.047 \text {. }
$$

When the $M$-matrix conditions are replaced by the stronger condition in (5.16), we obtain the estimate

$$
\Omega_{D}^{\text {H. }}: 3.598\left|p_{12}\right|+5.864\left|p_{21}\right|+1.886\left|p_{32}\right|<1 .
$$

We note that $\Omega^{W}$ is independent of $p_{32}$ as $\Omega$ in (6.3) is, and in this sense, is superior to the closed regions in (6.5) and (6.7). However, a further majorization as in (5.16) eliminates this advantage, as can be observed from (6.12).

We also note that each of the estimates in (6.5)-(6.7) can be further expanded by repeated application of the robustness analysis to a modified system obtained by moving the nominal system to a point on the boundary of the robustness region and redefining the perturbation parameters accordingly. However, since this process destroys the subsystem versus interconnection structure, it may not be suitable for interconnected systems.

We finally note that a scaling of the perturbation parameters and the corresponding perturbation matrices may be useful in obtaining improved robustness bounds, as noted in [10].

\section{REFERENCES}

[1] R. E. Kalman and J. E. Bertram, "Control system analysis and design via the second method of Lyapunov," Trans. Amer. Soc. Mech. Eng.J. Basic Eng., vol. 82, pp. 371-393, 1960.

[2] R. V. Patel and M. Toda, "Quantitative measures of robustness for multivariable systems," in Proc. Joint Automat. Contr. Conf., San Francisco, CA, 1980, paper TP8-A.

[3] R. K. Yedavalli, "Improved measures of stability of robustness for linear state space models," IEEE Trans. Automat Contr., vol. AC-30, pp. 577-579, 1985.

[4] R. K. Yedavalli and Z. Liang, "Reduced conservatism in stability robustness bounds by state transformation," IEEE Trans. Automat Contr., vol. AC-31, pp. 863-866, 1986.

[5] K. Zhou and P. P. Khargonekar, "Stability robustness bounds for linear state space models with structured uncertainty," IEEE Trans. Automat Contr., vol. AC-32, pp. 621-623, 1987.

[6] M. E. Sezer and D. D. Šijak, "Robust stability of discrete systems," Int. J. Contr., vol. 48, pp. 2055-2063, 1988.
[7] E. Yaz, "Deterministic and stochastic robustness measures for discrete systems," IEEE Trans. Automat Contr., vol. 33, pp. 952-955, 1988.

[8] S. R. Kolla, R. K. Yedavalli, and J. B. Farrison, "Robust stability bounds on time-varying perturbations for state-space models of linear discrete-time systems," Int. J. Contr., vol. 50, pp. 151-159, 1989.

[9] J. W. Brewer, "Kronecker products and matrix calculus in system theory," IEEE Trans. Circuits Syst., vol. CAS-25, pp. 772-780, 1978.

[10] M. E. Sezer and D. D. Šiljak, "A note on robust stability bounds," IEEE Trans. Automat Contr., vol. 34, pp. 1212-1214, 1989.

[11] M. Araki, "Stability of large-scale systems: Quadratic order theory of composite-system method using $M$-matrices," IEEE Trans. Automat Contr., vol. AC-23, pp. 129-142, 1978.

[12] D. D. Šiljak, Large-Scale Dynamic Systems. New York: North-Holland, 1978 .

\section{Design of Robust Controllers for Time-Delay Systems}

\author{
Magdi S. Mahmoud and Naser F. Al-Muthairi
}

Abstract-The problem of stabilizing linear dynamical time-delay systems subject to bounded uncertainties is investigated. Two memoryless feedback controllers are considered. It is established that when the matching conditions are met and certain bounding relations are satisfied, then the linear controller renders the zero-response of the system asymptotically stable. Saturation-type controllers are shown to guarantee that all system responses are uniformly ultimately bounded.

\section{INTRODUCTION}

A major problem in the analysis of linear dynamical systems with time-delay is related to their stabilization using linear feedback with or without memory. Several results are readily available in the literature; see [1]-[6] and the references cited therein. Some of the results have been successfully extended to include the effect of bounded uncertainties [7]-[10]. Preliminary investigations on discrete-time systems with state delay are found in [11]-[13]. A thorough review of the major past works of the deterministic approach to uncertain system based on the constructive use of Lyapunov function can be found in [14], [15]. If the uncertainty fits a certain characterization (often termed the matching condition), then a class of feedback controllers can be designed based only on the upper bound of the uncertainty [16]. When dealing with time-delay systems, it turns out [7], [9] that an additional assumption is needed to guarantee smooth behavior of the closed-loop state trajectories.

Perhaps the work of Thowsen [7] and $\mathrm{Yu}$ [8] were among the early investigations to include the effect of bounded uncertainties on dynamical systems with time delay. The results of [7] required a bounding assumption to hold for all possible solutions not just the nominal. This restriction was relaxed in [9] and applied to river pollution control. Linear uncertain systems with state delay were treated in [8] and the stabilizing controller had limited gain adjustment.

This work adds to the further development of stabilizing controllers for continuous-time uncertain systems with state delay. The major

Manuscript received July 21, 1992; revised December 4, 1992 and April 15, 1993. This work was supported by Kuwait University Research Administration under Grant Number EE-049.

The authors are with the Department of Electrical and Computer Engineering, Kuwait University, Safat, 13060 Kuwait.

IEEE Log Number 9216455 . 